\title{
Una mirada crítica a los modelos teóricos sobre educación STEAM integrada
}

\author{
Jairo Ortiz-Revilla ${ }^{1}$. \\ Raquel Sanz-Camarero ${ }^{1} \mathrm{O}$ \\ Ileana M. Greca ${ }^{1}$. \\ ${ }^{1}$ Universidad de Burgos (UBU), España
}

Resumen. La sociedad cambia rápidamente pero el sistema educativo no está siguiendo un camino paralelo, generándose una urgente necesidad de renovación educativa. En este sentido, la educación integrada de ciencias, tecnología, ingeniería, artes y matemáticas (i-STEAM) representa un enfoque educativo en plena expansión, que se postula alineado con las necesidades educativas de la sociedad; prueba de ello es el continuo incremento de publicaciones en esta línea de investigación. Existe una clara predominancia de estudios empíricos sobre este enfoque, mientras que su fundamentación teórica está menos desarrollada, lo que suscita un problema para definir su potencial educativo. En este estudio identificamos y revisamos los distintos modelos teóricos desarrollados para la i-STEAM publicados en revistas de alto impacto con el objetivo de realizar una reflexión crítica y de evaluar el alcance real de este enfoque. Los resultados obtenidos evidencian que, si bien existen algunos trabajos que muestran aspectos teóricos relevantes, aún hay pocos con marcos teóricos que proporcionen una fundamentación robusta y holística (teniendo en cuenta aspectos epistemológicos, psicológicos y didácticos) para la i-STEAM. A la luz de estos resultados recalcamos la necesidad de seguir trabajando en el soporte teórico de este enfoque, que permita a los docentes implementarlo de manera efectiva. Palabras clave: i-STEAM; fundamentación teórica; revisión sistemática; revisión estudio por estudio.

\section{Um olhar crítico sobre os modelos teóricos da educação STEAM integrada}

Resumo. A sociedade muda rapidamente, mas o sistema educacional não está seguindo um caminho paralelo, gerando uma necessidade urgente de renovação educacional. Neste sentido, a educação integrada de ciência, tecnologia, engenharia, artes e matemática (i-STEAM) representa uma abordagem pedagógica em plena expansão, alinhada com as necessidades educacionais da sociedade. Prova disso é o aumento contínuo das publicações nesta linha de pesquisa. Há uma clara predominância de estudos empíricos sobre esta abordagem, embora a sua fundamentação teórica seja menos desenvolvida, levantando um problema para a definição do seu potencial educacional. Neste estudo, identificamos e revisamos os diferentes modelos teóricos desenvolvidos para o i-STEAM publicados em revistas de alto impacto, com o objetivo de fazer uma reflexão crítica e avaliar o alcance real desta metodologia. Os resultados obtidos revelam que, embora existam alguns trabalhos que mostram aspectos teóricos relevantes, ainda há poucos marcos teóricos que oferecem uma fundamentação robusta e holística (considerando aspectos epistemológicos, psicológicos e didáticos) para o i-STEAM. De acordo com estes resultados, enfatizamos a necessidade de continuar trabalhando na base teórica desta abordagem, permitindo aos professores implementá-la eficazmente. Palavras-chave: i-STEAM; fundamentação teórica; revisão sistemática; revisão estudo por estudo.

A critical look at theoretical models on integrated STEAM education

Abstract. Society is changing rapidly and the educational system is not following a parallel path, generating an urgent need for educational renewal. In this sense, integrated science, technology, engineering, arts and mathematics education (i-STEAM) represents an educational approach in full expansion which is postulated to be aligned with the educational needs of society; proof of this is the continuous increase of publications in this line of research. There is a clear predominance of empirical studies on this approach, while its theoretical foundation is less developed, which raises a problem in defining its educational potential. In this study we identify and review the different theoretical models developed for i-STEAM published in high impact journals with the aim of making a critical reflection and assessing the real scope of this approach. The results obtained show that, although there are some papers that show relevant theoretical aspects, there are still few works with theoretical frameworks that could provide a robust and holistic foundation (considering epistemological, psychological and didactical aspects) for i-STEAM education. In light of these results, we emphasize the need to continue working on the theoretical support of this approach, which would allow teachers to implement it effectively. Keywords: i-STEAM; theoretical foundation; systematic review; study by study review. 


\section{Introducción}

Las carencias en la alfabetización integral y, con ello, científica, de la ciudadanía se visibilizan como un objeto recurrente de investigación, principalmente desde la investigación educativa. El estancamiento del desarrollo del alumnado en este sentido desde las primeras etapas educativas se atribuye, en buena medida, a la herencia dejada por la enseñanza tradicional (European Commission, 2007; Osborne y Dillon, 2008), cuestión en la que parece existir un consenso en la literatura. Entre otros aspectos, la clase tradicional y expositiva ha sido señalada como una de las mayores limitaciones del sistema educativo actual, siendo necesaria una renovación educativa acorde a los nuevos tiempos. Al respecto, el surgimiento de las denominadas metodologías activas ha significado dar un paso al frente en el camino hacia cambio, reportando resultados positivos y alentadores, como es el caso, por ejemplo, de la metodología de indagación (Aguilera et al., 2018; Romero-Ariza, 2017). Sin embargo, son cada vez más los autores que también refieren explícitamente las limitaciones que la enseñanza tradicional implica por su tratamiento compartimentado y aislado de los contenidos curriculares, alertando de la importancia de la integración disciplinar para un proceso de enseñanza-aprendizaje significativo (Bybee, 2013; Connor et al., 2015; Develaki, 2020; National Research Council [NRC], 2014, entre otros). Es desde de esta vertiente donde surge el abordaje educativo de la i-STEAM o educación STEAM integrada.

El ideario de integración disciplinar puede remontarse hasta Dewey (1859-1952) y ha sido recurrente en los debates y movimientos de reforma educativa desde el siglo pasado, en los cuales se han empleado argumentos psicológicos, epistemológicos y pedagógicos. En este momento, la integración parece ser liderada por la i-STEAM, la cual se postula como uno de los enfoques educativos con mayores expectativas para la mejora del desarrollo integral del alumnado a lo largo de las diferentes etapas educativas. De hecho, cada día surgen más publicaciones, llevadas a cabo desde multitud de contextos y lugares del mundo, que presentan intervenciones STEAM, propuestas, secuencias, actividades, etc. Sin embargo, así como estas publicaciones de carácter empírico se acumulan reportando beneficios en diferentes sentidos (Ata Aktürk y Demircan, 2017; Kang, 2019), existe una carencia de reflexiones teóricas profundas, meditadas y detalladas sobre la fundamentación teórica de la i-STEAM, cuestión que ya ha sido advertida por algunos autores (Aguilera y Ortiz-Revilla, 2021; McComas y Burgin, 2020; Millar, 2020; Zeidler, 2016).

Para evaluar el alcance real de la i-STEAM, resulta imprescindible reflexionar explícitamente sobre las cuestiones teóricas relacionadas con este enfoque, los fundamentos psicológicos y didácticos que sustentan su aplicación y sus eventuales beneficios, así como los posicionamientos epistemológicos y axiológicos a partir de los cuales se plantean (Ortiz-Revilla et al., 2020, Reynante et al., 2020). Solo de este modo se podrá aprovechar su potencial educativo.

Por ello, el objetivo de este estudio es realizar una reflexión crítica sobre los distintos modelos teóricos desarrollados para la i-STEAM y evaluar el alcance real de este enfoque. 


\section{Encuadre teórico}

Han pasado poco más de tres décadas desde la aparición del acrónimo inglés STEM (Science, Technology, Engineering and Mathematics) en el seno de la National Science Foundation (NSF). En sus inicios, y encuadrado en la preocupación de Estados Unidos por fomentar el aumento de mano de obra cualificada en las áreas científico-tecnológicas (Sanders, 2008), STEM emergió como un acrónimo de fácil memorización para referir a los planes de estudio relacionados con las cuatro disciplinas que lo componen y luego se empleó para describir los proyectos sobre tales disciplinas financiados por la NSF. Así, STEM se ha venido utilizando como una etiqueta genérica para mencionar cualquier evento, política, programa o práctica que implique a una o más de las disciplinas que lo componen (Bybee, 2010), convirtiéndose en un acrónimo polivalente y, sobre todo, popular. Tan popular, que ha dado lugar a lo que Sanders (2008) ha definido como STEMmanía: casi cualquier cosa excitante y nueva en educación se califica (erróneamente) como educación STEM (por ejemplo, el uso de robots).

En el transcurso de este camino ha confluido la noción de integración disciplinar, dando lugar a toda una amalgama de interpretaciones educativas de STEM (Breiner et al., 2012; Ritz y Fan, 2015) y, por tanto, a un significado aún ambiguo (MartínPáez et al., 2019). Este enfoque integrador, que transciende al significado primitivo de STEM, fue denominado educación STEM integrada (Kelley y Knowles, 2016), y supuso el germen de una línea de investigación y práctica educativa a la cual, más recientemente, se incluyeron las artes, dando lugar al acrónimo STEAM. Cabe destacar que las artes no quedan circunscriptas a la música, la plástica o la literatura, sino que "incluyen áreas como, por ejemplo, la sociología, la psicología, la historia, las bellas artes, la filosofía e, irónicamente, la educación" (Zeidler, 2016, p.17).

La estructura del enfoque STEAM comenzó a desarrollarse hace algo más de una década con el objetivo de alcanzar una educación holística e integradora, capaz de adaptarse a las numerosas combinaciones disciplinares que conforman las diferentes direcciones que persiguen las personas en la sociedad (Yakman, 2008). Esta ampliación del abanico disciplinar se considera un avance significativo con respecto a su predecesora, afirmándose que centrarse en STEM sin las "Artes", excluye necesariamente áreas importantes que informan y contextualizan la ciencia.

Así, se ha argumentado que la i-STEAM combina el trabajo estético y analítico característico de los modos de pensamiento de las artes y las ciencias y puede conducir a un aprendizaje transversal y sostenido (Bequette y Bequette, 2012). Un elemento que aparece reiteradamente desde las primeras conceptualizaciones sobre los abordajes i-STEAM es su carácter transdisciplinar y, en ese sentido, ligado a una postura epistemológica que entiende que la resolución de los problemas a los que nos enfrentamos como sociedad no pueden ser abordados mediante un enfoque exclusivamente disciplinar (Herro y Quigley, 2017). Por ello, varios autores defienden que "el objetivo de este enfoque es preparar a los estudiantes para resolver los problemas más acuciantes del mundo mediante la innovación, la creatividad, el pensamiento crítico, la comunicación eficaz, la colaboración y, en última instancia, los nuevos conocimientos" (Quigley y Herro, 2016, p.410). De este modo, la i-STEAM parece implicar 
una formación cuya finalidad es el desarrollo competencial integral del alumnado para su acción en la sociedad (Greca et al., 2021; Ortiz-Revilla, 2020; Ortiz-Revilla et al., 2018; Ortiz-Revilla, Greca y Meneses-Villagrá, 2021).

\section{Preguntas de investigación}

Ante este panorama, nos planteamos las siguientes preguntas de investigación:

- ¿Cuáles son las características de los modelos teóricos desarrollados para la i-STEAM?

- ¿Qué carencias es posible detectar en esos modelos en cuanto a su utilidad para una implementación y evaluación efectivas de la i-STEAM?

\section{Metodología}

Para dar respuesta a las preguntas de investigación anteriores, realizamos una revisión de la literatura con dos fases diferenciadas. En primer lugar, se llevó a cabo una revisión sistemática para recuperar los artículos que presentaban modelos teóricos sobre i-STEAM; concretamente, el proceso de selección se diseñó de acuerdo con la Declaración PRISMA para informar revisiones sistemáticas (Moher et al., 2009). En segundo lugar, se llevó a cabo una revisión bibliográfica "estudio por estudio" (Creswell y Guetterman, 2019) para efectuar su análisis en profundidad.

\subsection{Procedimiento de selección de los artículos}

Para la revisión sistemática, se llevó a cabo una búsqueda en las bases de datos Web of Science (WOS) y Scopus. La clave de búsqueda introducida consistió en la introducción de los términos STEAM y educat* OR teach* OR learn* en el campo topic, recuperando todos los documentos que presentaban estos términos bien en su título, resumen o palabras clave.

La búsqueda realizada en WOS reportó 1535 resultados y la ejecutada en SCOPUS 3054 resultados que, tras refinar mediante el filtro tipo de documento para incluir solo artículos, se redujo a 864 y 1339 resultados, respectivamente. Continuando con el refinado se empleó el filtro año de publicación para incluir solo los trabajos publicados entre 2008 (año en que, como hemos comentado, nació el acrónimo STEAM) y 2021 (ambos inclusive), el cual redujo a 729 los artículos procedentes de WOS y a 932 de SCOPUS que, tras un último refinado mediante el filtro idioma para incluir solo los artículos en inglés o español se redujeron a 697 de WOS y 841 de SCOPUS. De este total de 1538 artículos, se eliminaron 447 duplicados.

A continuación, se procedió a leer el título, el resumen y las palabras clave de los 1091 artículos filtrados, eliminándose 608 por no pertenecer a la temática de estudio (criterio 1 de exclusión) y 449 por no presentar un modelo teórico sobre i-STEAM (criterio 2 de exclusión). De este modo, quedaron 34 artículos que parecían presentar un modelo teórico, los cuales se leyeron completamente, descartándose 20 de ellos por no presentar una explicación del modelo teórico (criterio 3 de exclusión) y 5 más debido a que, si bien presentaban un modelo teórico, la información aportada era insuficiente para su análisis (criterio 4 de exclusión). Por tanto, quedaron 9 artículos para la revisión final. 
Posteriormente, se realizó la revisión estudio por estudio de los artículos finales. Todo este proceso se muestra con un diagrama de flujo (Figura 1).

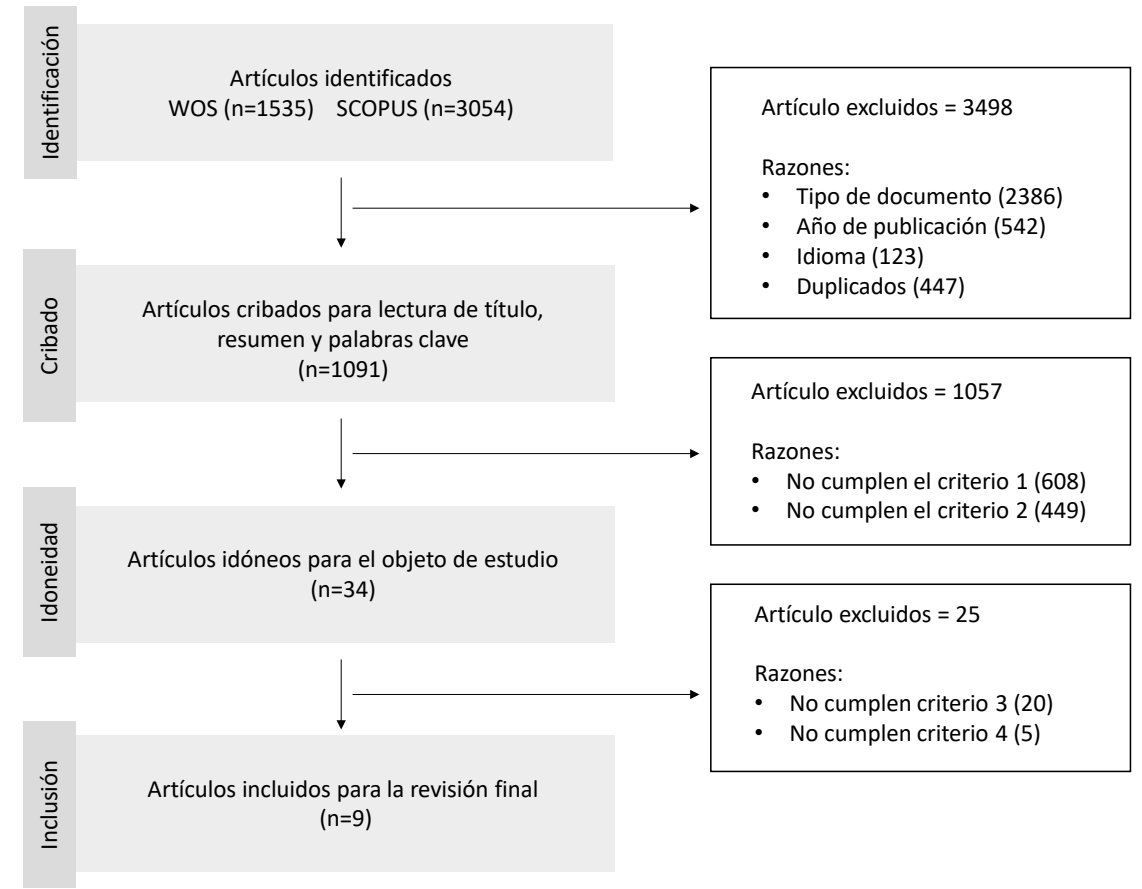

Figura 1. Diagrama de flujo del procedimiento de selección de artículos.

Fuente: elaboración propia.

\section{Resultados}

Los resultados se presentan divididos en dos partes. En la primera, se realiza una descripción de los parámetros generales del corpus bajo estudio y, en la segunda, se presenta la revisión en profundidad, donde su muestran las características centrales de cada modelo teórico propuesto.

\subsection{Descripción general de los estudios}

La Tabla 1 muestra un conjunto de parámetros de los artículos seleccionados: autores del estudio y año de su publicación, revista en la que está publicado el estudio, países de desarrollo, etapas educativas a las que se enfoca el modelo, tipo de integración disciplinar, objetivos de la i-STEAM, presencia de algún ejemplo de su aplicación práctica y evaluación de los resultados obtenidos tras su aplicación. 


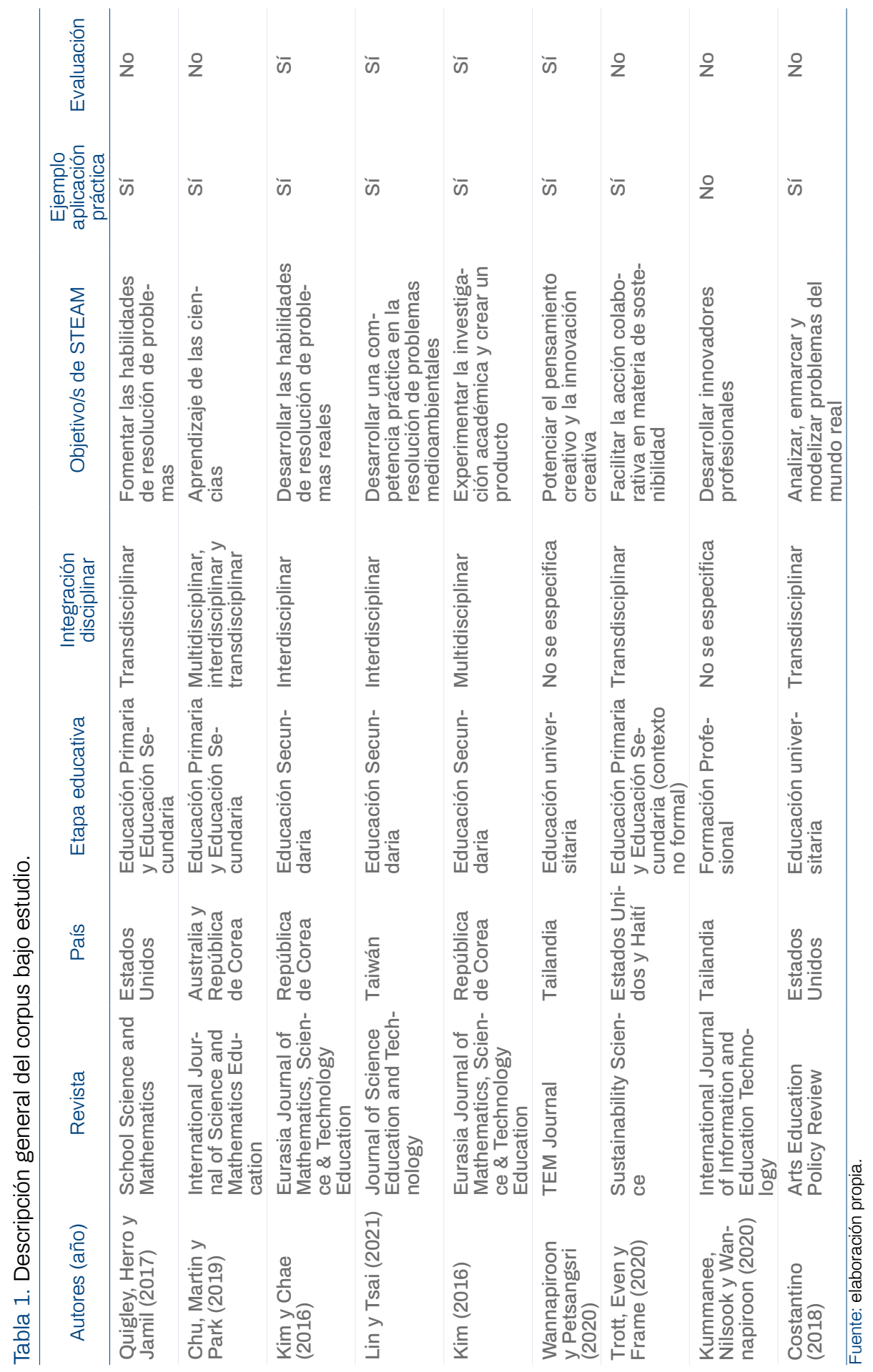


Como se puede observar, los estudios se han desarrollado en los últimos seis años, por autores que pertenecen a centros de investigación de Estados Unidos, Australia y de países del sudeste asiático. Los modelos abarcan todas las etapas educativas excepto la Educación Infantil, con un fuerte énfasis en la transdisciplinariedad. También se observa que la mayoría de estudios postula como objetivo de los abordajes i-STEAM el fomento de habilidades para la resolución de problemas del mundo real, entre ellos, los problemas relacionados con la sostenibilidad o el medio ambiente. Aunque la amplia mayoría de los estudios muestran ejemplos para la aplicación del modelo que plantean y lo llevan efectivamente a la práctica, más de la mitad de ellos no reporta los resultados obtenidos tras su aplicación.

\subsection{Revisión en profundidad}

A continuación, se presenta la revisión en profundidad estudio por estudio, donde se comienza por aclarar el propósito de cada estudio y se extraen los aspectos de relevancia estructural de cada modelo de i-STEAM planteado. Dado que resulta aclaratorio el uso de imágenes que sintetizan los modelos planteados, para aquellos casos en los que se proporciona y se ha considerado pertinente, se ha recogido también la imagen original y se ha traducido al español, manteniendo la estética del original (y en los casos necesarios el idioma original), para esta revisión.

El propósito de Quigley et al. (2017) es proponer un modelo conceptual para STEAM que proporcione a los educadores una vía para comprender y poner en práctica una instrucción STEAM eficaz. Su modelo está organizado en dos dominios: el contenido instructivo y el contexto de aprendizaje, abarcando un total de seis dimensiones esenciales. Por una parte, el contenido instructivo incluye las dimensiones del material didáctico de partida, la integración disciplinar y las habilidades para la resolución de problemas. La primera dimensión plantea que el punto de partida de las múltiples disciplinas debe ser problemas del mundo real (incluyendo conceptos, métodos y enfoques). En esta dimensión, también se indica cómo esos problemas deben posibilitar los objetivos de aprendizaje atendiéndose a cuatro criterios: la instrucción centrada en el problema, el propósito del contenido, la alineación de los estándares y la consideración de las disciplinas. La segunda dimensión destaca que el profesorado debe presentar el material de las diferentes disciplinas o áreas de contenido (ciencias, tecnología, ingeniería, artes y matemáticas) de forma clara y conectada, considerándose la conexión de los contenidos, las estrategias de enseñanza y la síntesis entre disciplinas. La tercera dimensión representa la forma en que el profesorado debe fomentar el desarrollo de las habilidades cognitivas, de interacción y creativas necesarias para la resolución eficaz de problemas. Por otra parte, el contexto de aprendizaje incluye las dimensiones de enfoques pedagógicos, prácticas de evaluación y participación equitativa. La primera de estas dimensiones contempla el modo en que el profesorado estructura el entorno del aula, las tareas y los recursos para facilitar el aprendizaje, creando entornos ricos para la realización de indagaciones en múltiples dominios, que integren la tecnología. La segunda se enfoca al proceso iterativo de perfeccionamiento de la instrucción y de la evaluación del aprendizaje utilizando múltiples formas de datos en un contexto real e implicando la alineación auténtica, la retroalimentación periódica, los ajustes basados en datos y la reflexión del alumnado. La tercera dimensión cubre el modo en que el aula facilita 
el acceso y la participación en el aprendizaje del alumnado con atención específica a las capacidades y recursos, contemplando la relevancia de la tarea, la diversidad, la responsabilidad y la elección del estudiante. Los autores proporcionan algunos escenarios para llevar a la práctica su modelo, sin embargo, existe una ausencia de aplicación real y de evaluación de resultados.

Chu et al. (2019) proponen un marco teórico para que los docentes desarrollen un programa STEAM encaminado a mejorar la enseñanza y el aprendizaje de las ciencias en un contexto intercultural. Contemplan tres elementos, que reúnen aspectos epistemológicos, psicológicos y metodológicos. En coherencia con su objetivo intercultural, se basan en el socioconstructivismo, asumiendo que los sujetos construimos colectivamente conocimiento, interactuando de manera colaborativa con otros y con el entorno, aspecto que es reforzado por su adopción del aprendizaje situado como postura psicológica, que ubica al aprendizaje como producto de la actividad, el contexto y la cultura en que se desarrolla. Desde el punto de vista metodológico, adoptan el modelo cíclico de instrucción 5E aplicado a la enseñanza basada en la indagación, un enfoque de aprendizaje cíclico que guía a los estudiantes a través de actividades que contemplan cinco etapas: participar, explorar, explicar, elaborar y evaluar. Así, una típica lección STEAM comienza con la etapa de participación, donde el compromiso se consigue haciendo que los estudiantes vean o experimenten un evento o un fenómeno en el que aparezca el concepto científico que se quiere abordar. Es decir, se parte de una situación, real o creada por los docentes, que debe ser necesariamente vivenciada, donde los estudiantes exploran y comparten. A continuación, en la fase de exploración, se procede a establecer discusiones en pequeños grupos para explorar conceptos a través de actividades prácticas que conducen a la generación de preguntas de indagación como, por ejemplo, ¿por qué? o ¿cómo? En respuesta a estas preguntas los estudiantes crean modelos explicativos que comparten con otros estudiantes en la etapa de explicación, redefiniéndose y perfeccionándose de manera colaborativa en la etapa de elaboración para, finalmente, ser evaluados con un modelo preciso en la última etapa de evaluación. Los autores realizaron una aplicación práctica de su modelo en cuatro colegios de Educación Primaria y dos de Educación Secundaria de Australia y República de Corea; no obstante, no reportan la evaluación en su manuscrito.

El propósito de la investigación de Kim y Chae (2016) es desarrollar un programa STEAM con la utilización elementos musicales tradicionales para diseñar una propuesta educativa convergente. Los autores proponen un marco de referencia que adhiere a tres pasos básicos propuestos por la Fundación Coreana para el Avance de la Ciencia y la Creatividad (Korea Foundation for the Advancement of Science and Creativity [KOFAC], 2012) (véase Figura 2): la presentación de la situación, el diseño creativo y el toque emocional, utilizados para potenciar las habilidades de resolución de problemas del mundo real en el alumnado. Estos elementos proporcionan experiencias de aprendizaje en las que convergen conocimientos y procesos relacionados con diversas áreas científico-tecnológicas, que impulsan a los estudiantes a resolverlas por iniciativa propia. Dentro del primer paso, según los autores, es importante que el alumnado reconozca que el problema está conectado con sus vidas y lo pueda relacionar con el mundo real. En el segundo paso, se anima a los estudiantes a resolver 
el problema de forma creativa, buscando sus propias soluciones. Además, el propósito de este paso es desarrollar tanto la creatividad como las habilidades de comunicación a través de una actividad de aprendizaje cooperativo. Por último, se busca ampliar el dominio afectivo del objetivo educativo y se subraya la importancia del corazón al experimentar y explorar una situación de aprendizaje, ayudando a los estudiantes a desarrollar la percepción, la expresión y la simpatía. Este programa se aplicó con 26 estudiantes de undécimo grado, cuyos resultados apuntan a que reconocieron el significado, la necesidad y la potencialidad de STEAM como un procedimiento de resolución de problemas, además de aumentar su alfabetización en estas áreas.

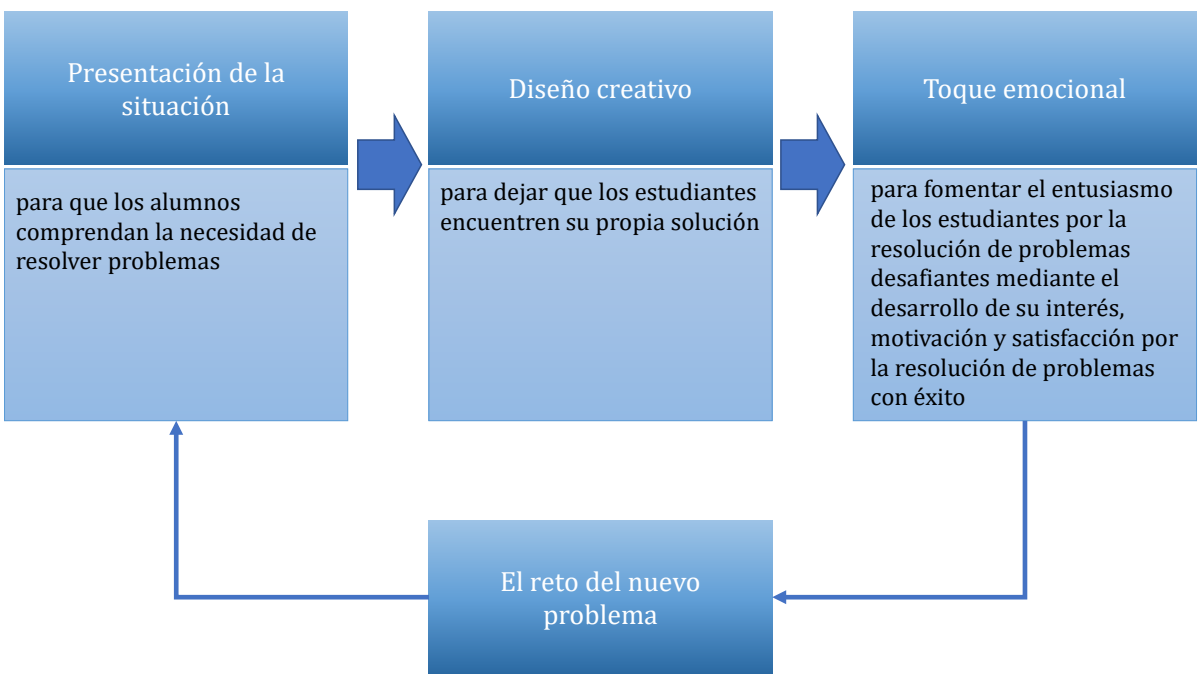

Figura 2. Marco de referencia para STEAM propuesto por KOFAC (2012).

Fuente: Adaptada de "The development and application of a STEAM programbased on traditional Korean culture", de H. Kim y D-H. Chae, 2016, Eurasia Journal of Mathematics, Science \& Technology Education, 12(7), p. 1927 (https://doi.org/10.12973/eurasia.2016.1539a). CC BY 4.0.

Lin y Tsai (2021) crean un modelo pedagógico (véase Figura 3) con el objetivo de implementar planes de estudio STEAM interdisciplinares, que abordan las ciencias (biología, química y ciencias de la tierra), la tecnología (biotecnología, tecnología de la información y tecnología verde), la ingeniería (tecnología viva, tecnología de prevención de desastres y aplicaciones electromecánicas), el arte (argumentación científica, dibujo científico, creatividad cultural y escritura científica) y las matemáticas (razonamiento lógico). Para ello, adoptan cinco estrategias pedagógicas: andamiaje, tutoría, participación, argumentación y modelado. Estas cinco estrategias parten de un andamiaje didáctico interdisciplinar, ofrecido por los docentes mediante cursos trasversales y recursos de aprendizaje (encuestas, bio indicadores, diseño de productos ecológicos, juegos y animaciones para el desarrollo de habilidades de modelización, etc.) para ayudar a los estudiantes a comprender y completar las tareas propuestas en cada fase del proyecto. Se usa una metodología basada en proyectos de corte ambiental, desarrollada en grupos. En este contexto, a cada grupo se le asigna un profesor que guía y aporta conocimientos para que puedan terminar el proyecto. A lo largo del plan de estudios se trabaja con experimentos, diseños y controversias socio-científicas 
para el desarrollo del pensamiento crítico y la comprensión de las repercusiones de la ciencia y la tecnología en la sociedad. Los estudiantes también desarrollan actividades de modelado en el proceso de resolver el proyecto. Finalmente, deben usar métodos científicos para completar los productos desarrollados en su proyecto y un informe final. Este modelo fue implementado con 114 estudiantes de Educación Secundaria en Taiwán, evaluándose una mejora de la competencia para realizar proyectos y de su motivación, así como una percepción positiva del modelo.

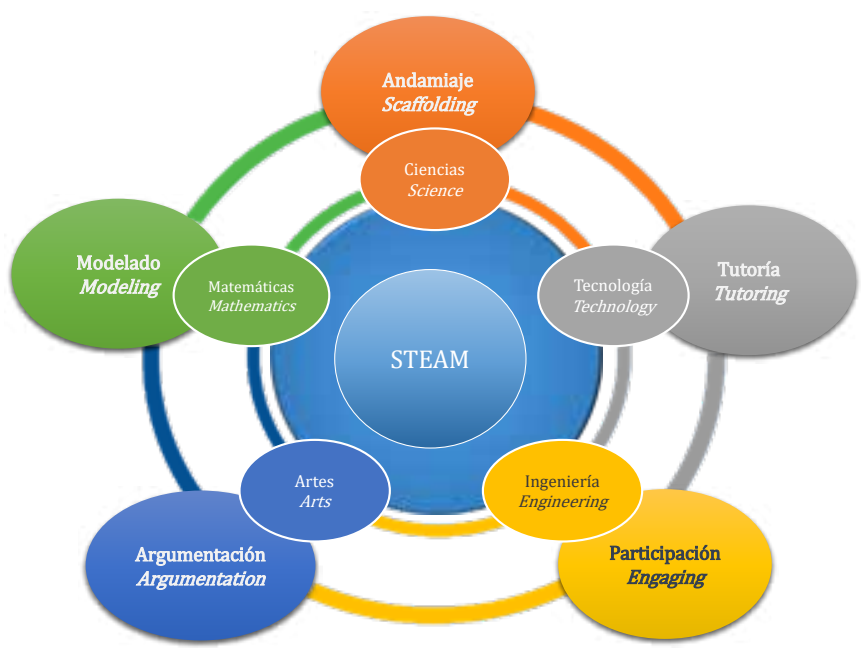

Figura 3. Modelo pedagógico STEAM propuesto por Lin y Tsai (2021).

Fuente: Adaptada de "The effect of a pedagogical STEAM model on students' project competence and learning motivation", de C-L. Lin y C-Y. Tsai, 2021, Journal of Science Education and Technology, 30(1), p. 114 (https://ijpe. penpublishing.net/arsiv/24). Copyright 2020 Springer Nature B.V. Reproducida con autorización.

El propósito principal de Kim (2016) es construir un modelo educativo convergente que utiliza contenidos científicos tradicionales coreanos como medio para conectar STEAM y las Humanidades. A través de un enfoque disciplinar paralelo, se aborda un tema que se desarrolla a través de ocho materias, conformando un estudio multidisciplinar. El autor compara este modelo con la estructura de una rueda, donde el tema central representa el eje; los estudios disciplinares corresponden a los ocho radios que engloban las cinco áreas de STEAM y las tres áreas adicionales de Humanidades; y el estudio multidisciplinar es la llanta (véase parte a de la Figura 4). De este modo, el alumnado ha de comenzar por adquirir un conocimiento completo del tema extraído de los contenidos. Posteriormente, selecciona uno de los ocho estudios disciplinares desde el que lleva a cabo su proyecto durante el primer periodo escolar de cinco meses, en el que aprende, al menos, los métodos de recogida de datos e información, de resumen y análisis de material diverso y de elaboración de informes. El segundo periodo escolar de otros cinco meses se enfoca a que cada equipo de estudiantes comunique los resultados de su investigación y se proceda a la fase de estudio multidisciplinar convergente. Dentro de esta fase, el alumnado desarrolla un producto a través de un proceso jerarquizado en cuatro niveles: datos, información, conocimiento y producto (véase parte b de la Figura 4). Así, experimenta la creación 
de productos mediante la convergencia de conocimientos, que va más allá de la simple combinación de los conocimientos de varios campos. Este modelo fue aplicado con 60 estudiantes en dos escuelas de Educación Secundaria de la República de Corea, detectándose niveles altos de satisfacción. El estudiantado consideró que la propuesta fue especialmente útil para la exploración de su trayectoria profesional, así como para la mejora de sus habilidades comunicativas y, en menor medida, para la mejora de su pensamiento lógico y de su logro académico.
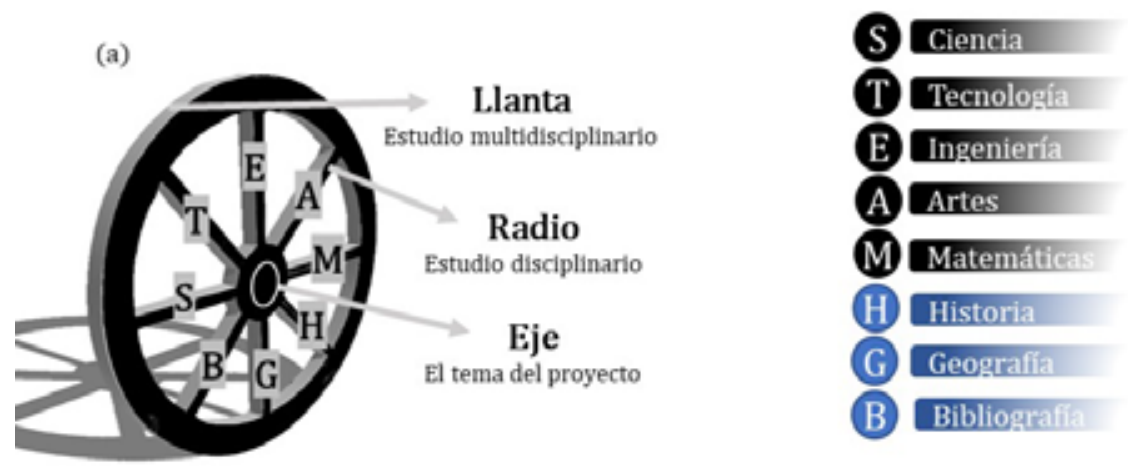

(a) Los ocho estudios disciplinarios del modelode la rueda (STEAM y HGB)

(b)

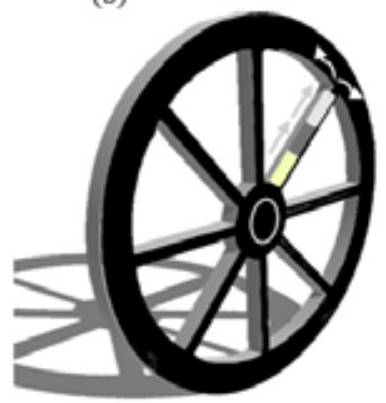

\author{
Producte \\ Al converger los conocimientos inducidos por los estudios de \\ las disciplinas, se desarrolla el estudio multidisciplinar \\ Conocimiento \\ CE Conocimiento Encontrado \\ CG Conocimiento Generado
}

Información IE Información Encontrada
IG Información Generada
Tema Datos
$\begin{aligned} & \text { DE Datos Encontrados } \\ & \text { Tema extraido de los contenidos de la ciencia tradicional }\end{aligned}$

(b) Los cuatroniveles de la etapade ejecución del proyecto $(\mathrm{D} \rightarrow \mathrm{I} \rightarrow \mathrm{C} \rightarrow \mathrm{P})$

Figura 4. Modelo de la rueda de Kim (2016).

Fuente: Adaptada de "The wheel model of STEAM education based on traditional Korean scientific contents", de P. W. Kim, 2016, Eurasia Journal of Mathematics, Science \& Technology Education, 12(9), p. 2356 (https://doi. org/10.12973/eurasia.2016.1263a). CC BY 4.0.

Wannapiroon y Petsangsri (2020) se proponen como objetivo principal desarrollar el modelo de STEAMificación en un entorno de aprendizaje de aula invertida para potenciar el pensamiento y la innovación creativos. Proponen un modelo que consta de seis componentes principales: instructor, alumno, contenido de aprendizaje, recurso en línea, infraestructura y entorno de aprendizaje de aula invertida (véase Figura 5). En este modelo, además, se plantean tres pasos a seguir: (1) preparación antes del experimento, que supone la orientación del alumnado por parte del instructor sobre cómo utilizar la tecnología para la mejora del aprendizaje; (2) actividad de 
aprendizaje STEAMificación en el entorno de aprendizaje de aula invertida, que hace uso de la gamificación, utilizando la mecánica y la dinámica del juego (basada en investigar, descubrir, conectar, crear innovación creativa y reflexionar) como elemento didáctico central; y (3) evaluación, que consta de dos aspectos clave, la habilidad de pensamiento creativo (con cuatro subhabilidades, originalidad, fluidez, flexibilidad y pensamiento de elaboración) y la innovación creativa (con cinco componentes, novedad y singularidad, resolución de problemas, eficiencia, posibilidades y coste). El modelo de STEAMificación se aplicó con 60 estudiantes del Programa de Tecnología Multimedia en una universidad de Tailandia; el grupo experimental obtuvo resultados más altos que los del grupo control en cuanto a la habilidad de pensamiento creativo y la calidad de la innovación creativa.

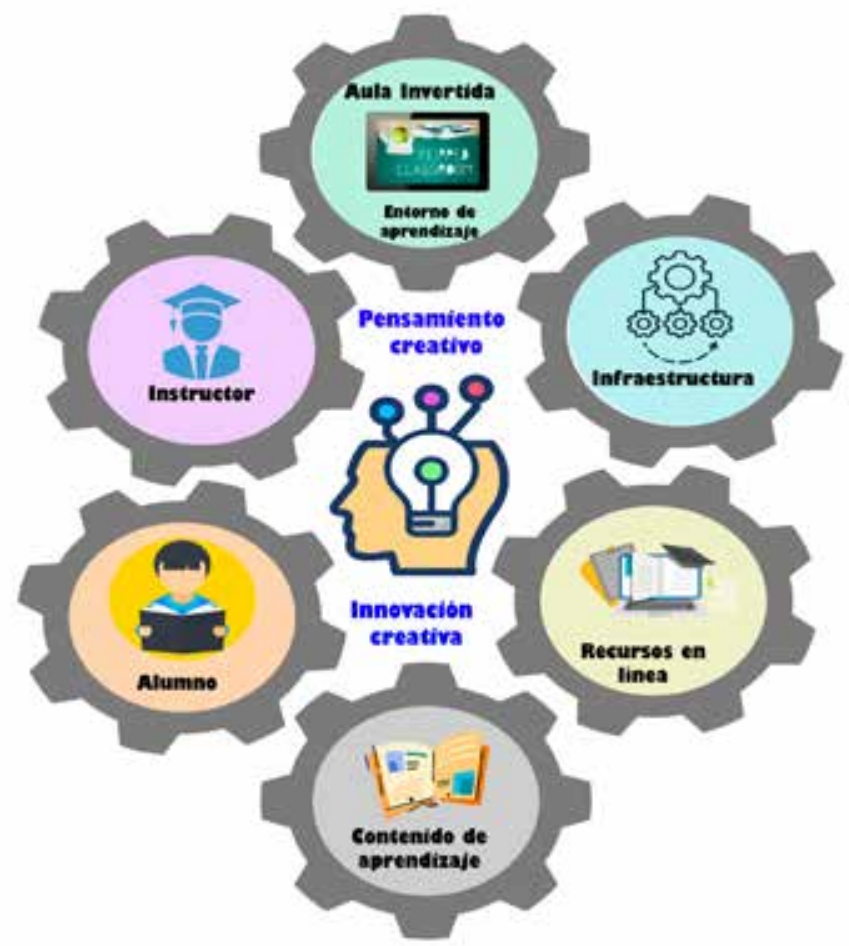

Figura 5. Componentes del modelo de STEAMificación de Wannapiroon y Petsangsri (2020).

Fuente: Adaptada de "Effects of STEAMification model in flipped classroom learning environment on creative thinking and creative innovation", de N. Wannapiroon y S. Petsangsri, 2020, TEM Journal, 9(4), p. 1651 (https://doi. org/10.18421/TEM94-42). CC BY-NC-ND 4.0.

Trott et al. (2020) buscan comprometer a los jóvenes para que imaginen un mejor futuro y actúen colaborativamente en favor de la sostenibilidad. Según expresan los autores, aunque muchas de las propuestas que incluyen el arte están más focalizadas hacia la competitividad, puede irse mucho más allá, siendo la integración del arte y las ciencias un elemento esencial para profundizar en la comprensión y el compromiso con los retos de la sostenibilidad. Por un lado, ofrece una vía para supe- 
rar algunas divisiones construidas e institucionalizadas (por ejemplo, emoción-razón y arte-ciencia), que parecen obstaculizar la transformación sostenible y, por la otra, posibilita ideas y métodos, que podrían originar nuevas respuestas para nuestros viejos problemas de sostenibilidad. Para ello, presentan un marco metodológico que integra las artes y las ciencias combinando tres elementos (véase Figura 6): (1) el aprendizaje transdisciplinar, que organiza la enseñanza y el aprendizaje en torno a la construcción de significados en el contexto de problemas o temas del mundo real y centrado en la comprensión de los retos de la sostenibilidad; (2) el proceso participativo, un enfoque colaborativo que reúne a investigadores y participantes para identificar, estudiar y abordar los problemas en los entornos comunitarios, centrado en el compromiso crítico con las realidades actuales no sostenibles y la planificación del cambio social. En la intersección de ambos procesos se encuentra (3) la acción colaborativa, un enfoque de acción comunitaria que implica trabajar juntos para la transformación de la sociedad hacia la sostenibilidad, centrada en generar activamente alternativas sostenibles en el ámbito local. Cabe destacar que, aunque de forma explícita, los autores no colocan los problemas como punto de partida de sus propuestas, son efectivamente los problemas de sostenibilidad cercanos al alumnado el punto para un planteamiento que envuelve una acción colaborativa dirigida por los jóvenes para la sostenibilidad. Este marco metodológico se aplicó a través de dos programas extraescolares, con 55 niños de 10 a 12 años en el oeste de Estados Unidos y con 21 de 8 a 14 años en el sur de Haití. Sin embargo, los autores no proporcionan una evaluación clara de los resultados obtenidos.

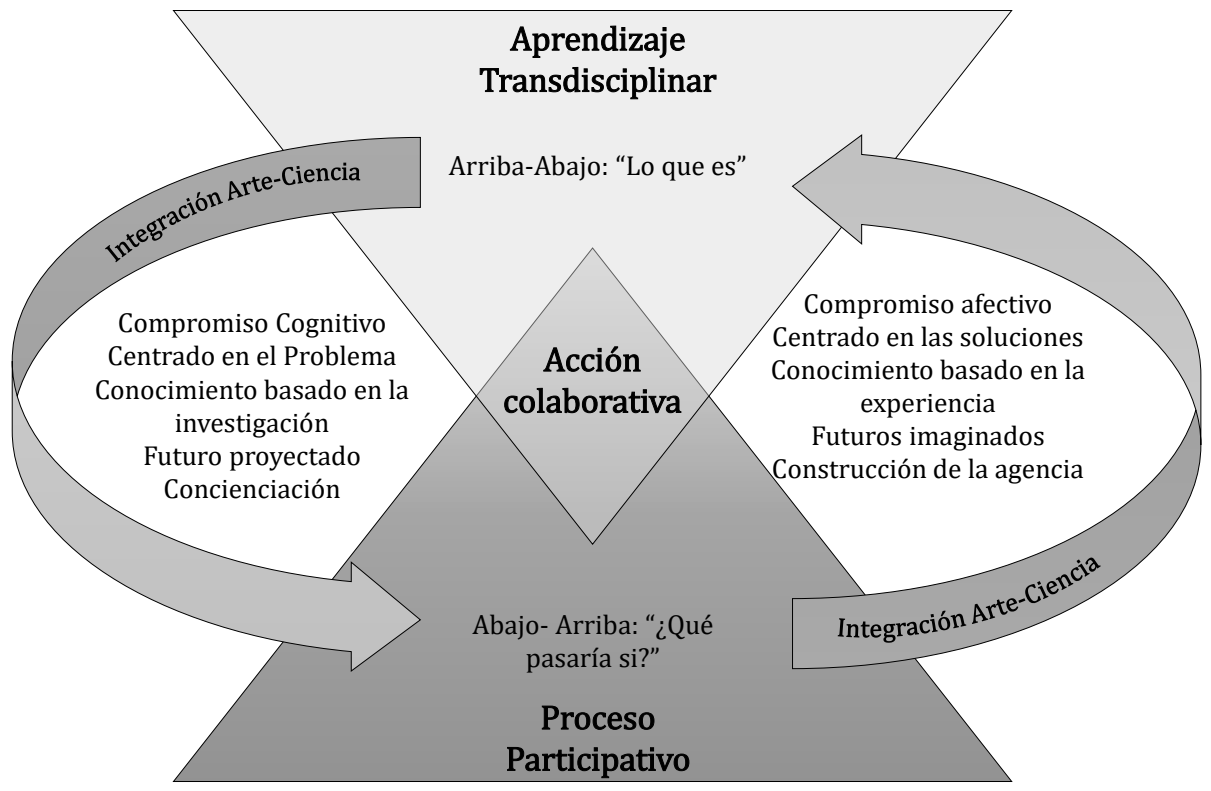

Figura 6. Marco metodológico de Trott et al. (2020).

Fuente:Adaptada de "Merging the arts and sciences for collaborative sustainability action: a methodological framework" de C. D. Trott, T. L. Even y S. M. Frame, 2020, Sustainability Science, 15(4), p. 1073 (https://doi.org/10.1007/ s11625-020-00798-7). CC BY 4.0. 


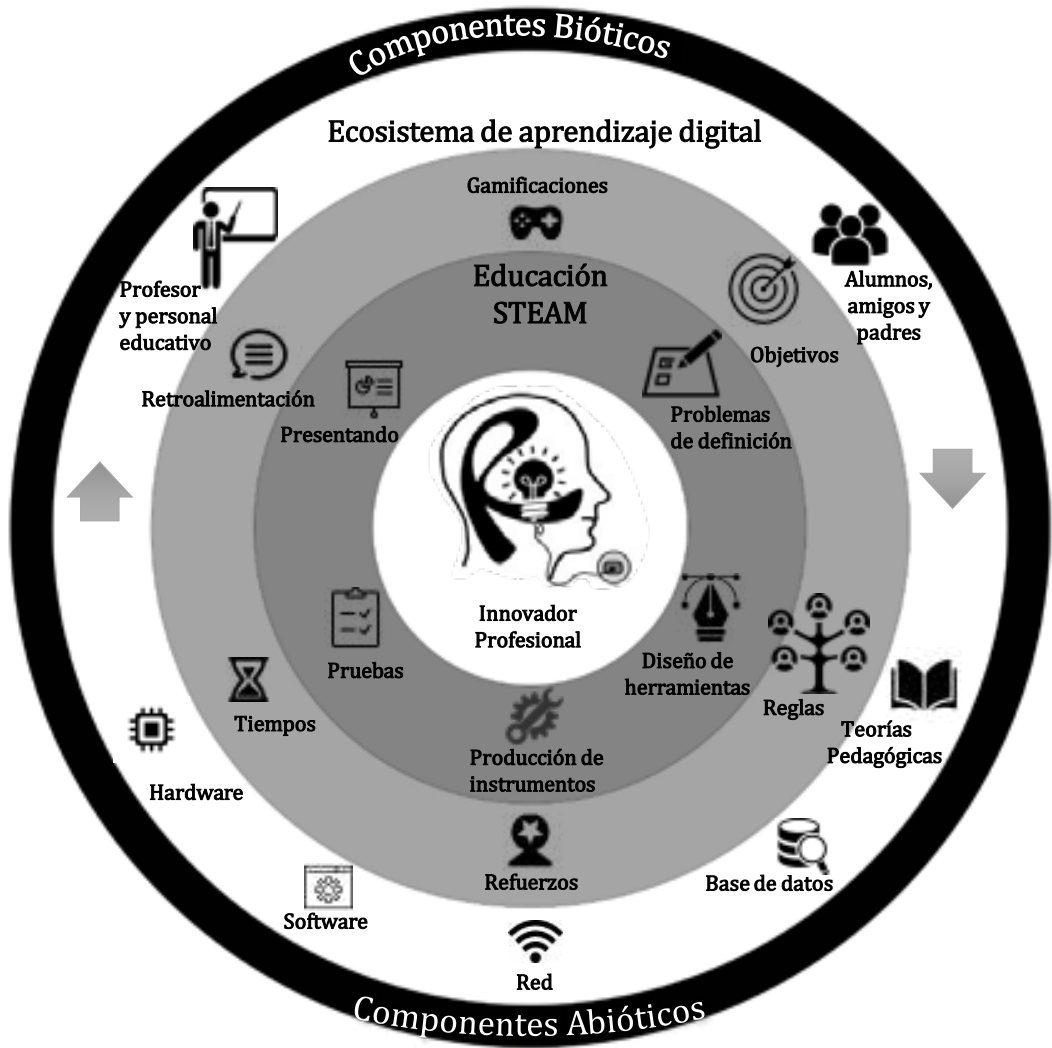

Figura 7. Modelo de ecosistema de aprendizaje digital propuesto por Kummanee et al. (2020).

Fuente: Adaptada de "Digital learning ecosystem involving steam gamification for a vocational innovator" de J. Kummanee, P. Nilsook y P. Wannapiroon, 2020, International Journal of Information and Education Technology, 10(7), p. 537 (https://doi.org/10.18178/ijiet.2020.10.7.1420). CC BY 4.0.

El principal propósito de Kummanee et al. (2020) es sintetizar el marco conceptual de un ecosistema de aprendizaje digital con gamificación STEAM para desarrollar vocaciones innovadoras, suponiendo que STEAM es un modelo de aprendizaje. Así, diseñan y desarrollan un modelo basado en la gamificación STEAM, las habilidades de innovación y el logro de aprendizaje. Este modelo consta de tres partes (véase Figura 7). La primera es el ecosistema de aprendizaje digital, formado por: el componente biótico, que incluye a los usuarios del sistema, como el profesorado y el personal educativo, los estudiantes, amigos y familiares; y el componente abiótico, que incluye el hardware, el software, la red, la base de datos y las teorías pedagógicas. Esta lógica propone el aprendizaje digital como un vínculo entre el componente biótico y el componente abiótico del sistema. Esto incluye al profesorado que transfiere conocimientos y facilita al alumnado la utilización de los componentes de los sistemas digitales de la manera más eficaz. La segunda parte es la gamificación STEAM. En este sentido, la realización de actividades de aprendizaje en el aula, mediante procesos educativos de aprendizaje centrados en la integración de la ciencia y el trabajo en equipo para 
desarrollar habilidades innovadoras, consta de cinco pasos: la definición del problema; el diseño de herramientas, es decir, el diseño de la solución el problema; la producción de instrumentos, esto es, la construcción de las herramientas de resolución del problema; el testeo o la interpretación del procedimiento de prueba y la mejora de los métodos de solución del problema; y la presentación de los métodos de solución. Y la tercera parte corresponde a los elementos de gamificación, que consisten en objetivos, reglas, refuerzo, tiempos y retroalimentación. Así, en la realización de las actividades de aprendizaje, los profesores utilizan los mecanismos de gamificación para estimular el interés y la diversión en el aula. En este caso, los autores no proporcionan ningún ejemplo llevado a la práctica ni su evaluación.

Por último, el objetivo de Constantino (2018) es esbozar un modelo transdisciplinar de estudios, basado en el proceso de investigación creativa y enmarcado en las pedagogías del arte y del diseño. La autora presenta los diversos componentes del modelo a través de un ejemplo de proyecto de investigación curricular que emplea herramientas de pensamiento de procesos típicos de la ingeniería y del arte. Se trata de un modelo robusto e iterativo, centrado en la definición y el perfeccionamiento del problema, en la exploración multimodal y material recurrente, en la elaboración crítica y en la presentación de ideas, con una crítica que se produce en múltiples momentos del proceso de investigación y en la exposición como un punto del ciclo que también puede generar un replanteamiento del problema y estimular nuevas investigaciones. Las 13 herramientas de pensamiento son: observar, imaginar, abstraer, reconocer patrones, formación de patrones, analogía, pensamiento corporal, empatía, pensamiento dimensional, modelar, jugar, transformar y sintetizar. Estas herramientas no se enseñan de forma aislada, sino que se aplican como formas de pensar en un proceso de resolución de problemas, donde el alumnado sintetiza su aprendizaje trabajando en equipos. Este modelo de investigación creativa, si bien no es específico para STEAM, según la autora, presenta un carácter transdisciplinar que hace hincapié en el aprendizaje basado en problemas y que valora intrínsecamente las pedagogías características de la educación artística y del diseño en relación sinérgica con una o más disciplinas STEM, pudiendo proporcionar oportunidades para la implementación de la i-STEAM. Este modelo fue aplicado con nueve estudiantes de la Licenciatura en Ingeniería Ambiental y diez estudiantes de Arte. Sin embargo, no se presenta una evaluación de sus resultados.

Para finalizar esta revisión en profundidad, en la Tabla 2 aparecen sintetizadas las dimensiones abordadas en cada modelo.

Tabla 2. Relación de dimensiones abordadas en los modelos teóricos.

\begin{tabular}{lcccc}
\hline \multicolumn{1}{c}{ Autores } & \multicolumn{3}{c}{ Dimensiones } \\
& Epistemológica & Psicológica & Didáctica & Metodológica \\
$\begin{array}{l}\text { Quigley, Herro y Jamil } \\
(2017)\end{array}$ & $\mathrm{x}$ & $\mathrm{x}$ & $\mathrm{x}$ \\
$\begin{array}{l}\text { Chu, Martin y Park } \\
(2019)\end{array}$ & $\mathrm{x}$ & $\mathrm{x}$ & $\mathrm{x}$ & $\mathrm{x}$ \\
Kim y Chae (2016) & & p* & $\mathrm{x}$ & $\mathrm{x}$
\end{tabular}


Dimensiones

Autores

$$
\text { Epistemológica Psicológica Didáctica Metodológica }
$$

Lin y Tsai (2021)

Kim (2016)

Wannapiroon y Petsangsri (2020)

Trott, Even y Frame (2020)

Kummanee, Nilsook y

Wannapiroon (2020)

$\begin{array}{ccc} & x & x \\ & x & x \\ x * & x & x \\ x & x & x \\ x & x\end{array}$

Costantino (2018)

${ }^{*} p$ (parcialmente abordada): la dimensión aparece en la descripción del modelo, aunque no se identifica un referencial teórico.

Fuente: elaboración propia.

\section{Discusión y conclusiones}

Como indicamos, la i-STEAM ha cobrado relevancia en el contexto de la urgente necesidad de renovación educativa. Desde la propuesta del famoso modelo de la pirámide de Yakman (2008), que establecía niveles de integración disciplinaria y estructuraba la naturaleza interactiva de las disciplinas (al considerar STEAM como la ciencia y la tecnología interpretadas a través de la ingeniería y las artes, todo basado en el lenguaje matemático) es apreciable la evolución que ha experimentado la fundamentación teórica de este enfoque.

Este desarrollo de modelos teóricos para la i-STEAM se ha llevado a cabo en los últimos años, lo que indica que se ha comenzado a atender la necesidad advertida en la literatura sobre la importancia de la fundamentación teórica de este enfoque (Aguilera et al., 2021; McComas y Burgin, 2020; Millar, 2020; Ortiz-Revilla et al., 2020; Reynante et al., 2020; Zeidler, 2016).

Es también claramente apreciable que los modelos teóricos propuestos provienen de países del sudeste asiático y de Estados Unidos, no encontrándose ningún modelo procedente de Iberoamérica o Europa, a pesar de la larga tradición en el área de la enseñanza de las ciencias y la tecnología en estas regiones. Esta cuestión resulta congruente con las diversas directrices políticas asiáticas y estadounidenses, que han propulsado propuestas STEAM en los últimos años. Al parecer, la investigación teórica sobre la i-STEAM ha emergido en tanto que las políticas educativas nacionales han atendido a este enfoque, y financiado numerosas investigaciones empíricas. Por ello, cabe esperar que en un futuro próximo la procedencia de los modelos teóricos se diversifique. Por ejemplo, algunos países, como España, comienzan a introducir enfoques integrados en el currículo de la educación obligatoria, así como los gobiernos y organizaciones civiles de varios países de Latinoamérica están impulsando la implementación de enfoques STEAM en Educación Primaria y Secundaria (Corfo y Fundación Chile, 2017; Espinal y Silveira, 2019). En el mismo sentido, es probable un aumento en el desarrollo de propuestas teóricas en Europa, dado que la Unión Europea está financiando diversos proyectos en esta línea. 
Las etapas de educación obligatorias son las más abordadas al fundamentar teóricamente STEAM, con una presencia mayor de la Educación Secundaria sobre la Educación Primaria, resultados que concuerdan con otras revisiones recientes sobre STEAM (Kwan y Wong, 2021). No obstante, también se han encontrado estudios enfocados a la Educación Universitaria y a la Formación Profesional. Todo esto parece indicar el interés existente por la implementación de la i-STEAM desde las etapas tempranas de escolarización. Si bien a menudo se piensa que la etapa de Educación Primaria podría presentar más facilidades desde el punto de vista organizativo a la integración disciplinar, parece que en la literatura esta cuestión está siendo superada, al transcender las posibles dificultades de integración disciplinar que se pueden presentar en la organización escolar tanto de la Educación Secundaria como de la Universitaria, en las que predomina el profesorado especialista en una materia.

En cuanto a la integración disciplinar, la mayoría de los estudios considera la multidisciplinariedad, la interdisciplinariedad y la transdisciplinariedad para la i-STEAM, ubicándose en los niveles más altos de integración (Gresnigt et al., 2014). Sin embargo, Quigley y Herro (2016) señalan que los enfoques multidisciplinares no se centran habitualmente en la resolución de problemas y argumentan sobre la necesidad de la adopción de enfoques inter y transdisciplinares para la i-STEAM. En este sentido, sin embargo, dada la polisemia inherente a lo que es multi, inter y transdisciplinar en los ámbitos epistemológico y educativo (Ortiz-Revilla, Greca y Arriassecq, 2021), hay que ser cautelosos. Quizás podemos acordar que todos los modelos aquí propuestos parten de problemas reales, vivenciados por el estudiantado y abordados con conocimientos y habilidades provenientes de diferentes disciplinas claramente identificadas, lo que implica una integración real. Es relevante destacar que, en relación con la integración de las artes, estas son consideradas en varios de ellos en un sentido amplio (liberal arts) y los modelos no caen en el reduccionismo de su consideración exclusivamente para el desarrollo de la creatividad (Aguilera y Ortiz-Revilla, 2021), como popularmente se señala y muchas veces se critica desde el ámbito académico.

Sobre los objetivos perseguidos con la i-STEAM, en la mayoría de casos se percibe la noción de resolución de problemas, bien indicada explícitamente o bien subyacente a otras cuestiones, por ejemplo, las relacionadas con la sostenibilidad. Así, son pocos los modelos que se alejan de esta esencia de STEAM, refiriendo, por ejemplo, al aprendizaje de las ciencias, a la potenciación del pensamiento creativo o al desarrollo de innovación profesional.

Un resultado que cabía esperarse en base a la producción científica sobre STEAM es que la mayoría de estudios presenta ejemplos de aplicación práctica del modelo planteado, aunque pocos son los que presentan una evaluación de dichos resultados. Dada la relativa novedad de los artículos encontrados, es de esperar que en los próximos años aparezcan publicaciones con estos resultados. Este aspecto es de especial importancia pues, si bien en este artículo analizamos fundamentaciones teóricas, los resultados empíricos son los verdaderamente determinan la validez y viabilidad de los modelos.

En cuanto a las dimensiones, todos los modelos incluyen la dimensión metodológica, es decir, aluden de un modo u otro a metodologías concretas para viabilizar en el aula el enfoque STEAM, aunque algunos con escasa información (véase Lin y 
Tsai, 2021; Wannapiroon y Petsangsri, 2020). Coherentemente, los autores proponen metodologías activas y centradas en el alumnado, como el aprendizaje basado en problemas, el aprendizaje basado en proyectos o la metodología de indagación. Prácticamente todos los modelos también incluyen la dimensión didáctica y explicitan las estrategias pedagógicas necesarias para la aplicación del modelo, por ejemplo, la gamificación, la argumentación o el aula invertida. Sin embargo, generalmente estas directrices cuentan con demasiados pasos o fases que pueden hacer complejo su entendimiento para trasladarlo al aula. Por otra parte, menos de la mitad de estudios aborda de forma explícita las dimensiones psicológica y epistemológica, siendo en algunos de ellos parcialmente abordadas. Dentro de la dimensión psicológica, relativa a las teorías adoptadas sobre el proceso de adquisición de conceptos y habilidades, aspecto fundamental para determinar el formato metodológico más adecuado, encontramos el aprendizaje situado, el aprendizaje colaborativo y la educación emocional. La dimensión epistemológica es la menos abordada, a pesar de su relevancia para comprender la naturaleza de la producción del conocimiento científico-tecnológico y, con ello, ayudar al desarrollo de una visión crítica en el alumnado. Esta ausencia podría significar posiciones acríticas por parte de los autores.

Por ello, consideramos de extrema importancia que los modelos sean claramente descriptos, incluyendo de forma explícita los supuestos en los que se fundamentan. Si no, su aplicación quedará reducida a sus contextos específicos, contribuyendo muy poco a que los docentes puedan implementarlos, dada su escasa formación sobre enfoques integrados en general y enfoques STEAM en particular (García-Carrillo et al., 2021). En este sentido, la reciente propuesta de un marco teórico para la i-STEM desarrollada por Ortiz-Revilla et al. (2021), que aborda explícitamente todas las dimensiones, podría ser adaptada para la i-STEAM.

En síntesis, parece plausible afirmar que, si bien hemos detectado en esta revisión carencias para la fundamentación teórica de la i-STEAM que deben ser subsanadas, existe bastante trabajo realizado en este sentido, con fundamentaciones que abarcan más de una dimensión, lo que por mucho tiempo no ha sucedido en abordajes con varias décadas de tradición en la didáctica de las ciencias como el uso de la Historia y la Filosofía de la Ciencia (Teixeira et al., 2012) o el abordaje de Ciencia, Tecnología y Sociedad (Zeidler et al., 2005). Esto demuestra el desacierto crítico de las visiones que afirman la inexistencia de esfuerzos al respecto que invalidaría la i-STEAM.

En esta misma dirección, cabe destacar que estos modelos muestran cómo es posible articular, considerando aportes psicológicos, didácticos y metodológicos, enfoques verdaderamente integrados que, independientemente de estar o no dentro de una línea STEAM, destacan como efectivos e indispensables para el desarrollo competencial del alumnado del sigo XXI (Drake y Reid, 2020; Little, 2012). 


\section{Referencias $^{1}$}

Aguilera, D., Lupiáñez, J. L., Vílchez-González, J. M., y Perales-Palacios, F. J. (2021). In search of a long-awaited consensus on disciplinary integration in STEM education. Mathematics, 9(6), 597. https://doi.org/10.3390/math9060597

Aguilera, D., Martín-Páez, T., Valdivia-Rodríguez, V., Ruiz-Delgado, A., Williams-Pinto, L., VílchezGonzález, J. M., y Perales-Palacios, F. J. (2018). La enseñanza de las ciencias basada en indagación. Una revisión sistemática de la producción española. Revista de Educación, 381, 259-284. http://doi.org/10.4438/1988-592X-RE-2017-381-388

Aguilera, D., y Ortiz-Revilla, J. (2021). STEM vs. STEAM education and student creativity: a systematic literature review. Education Sciences, 11(7), Artículo 331. https://doi.org/10.3390/ educsci11070331

Ata Aktürk, A., y Demircan, H. O. (2017). A review of studies on STEM and STEAM education in early childhood. Ahi Evran Üniversitesi Kırşehir Eğitim Fakültesi Dergisi (KEFAD), 18(2), 757776. https://bit.ly/3hUYz4D

Bequette, J. W., y Bequette, M. B. (2012). A place for art and design education in the STEM conversation. Art Education, 65(2), 40-47. https://doi.org/10.1080/00043125.2012.11519167

Breiner, J. M., Harkness, S. S., Johnson, C. C., y Koehler, C. M. (2012). What is STEM? A discussion about conceptions of STEM in education and partnerships. School Science and Mathematics, 112(1), 3-11. https://doi.org/10.1111/j.1949-8594.2011.00109.x

Bybee, R. W. (2010). Advancing STEM education: a 2020 vision. Technology and Engineering Teacher, 70(1), 30-35. https://bit.ly/2W4Fsh2

Bybee, R. W. (2013). The case for STEM education: challenges and opportunities. NSTA.

*Chu, H-E., Martin, S. N., y Park, J. (2019). A theoretical framework for developing an intercultural STEAM program for Australian and Korean students to enhance science teaching and learning. International Journal of Science and Mathematics Education, 17(7), 1251-1266. https://doi.org/10.1007/s10763-018-9922-y

Connor, A. M., Karmokar, S., y Whittington, C. (2015). From STEM to STEAM: strategies for enhancing engineering \& technology education. International Journal of Engineering Pedagogies, 5(2), 37-47. https://doi.org/10.3991/ijep.v5i2.4458

${ }^{*}$ Constantino, T. (2018). STEAM by another name: transdisciplinary practice in art and design education. Arts Education Policy Review, 119(2), 100-106. https://doi.org/10.1080/10632913.2017 .1292973

Corfo y Fundación Chile. (2017). Preparando a Chile para la sociedad del conocimiento: hacia una coalición que impulse la Educación STEAM. https://bit.ly/3zufT6v

Creswell, J. W., y Guetterman, T. C. (2019). Educational research: planning, conducting, and evaluating quantitative and qualitative research (6th ed.). Pearson.

Develaki, M. (2020). Comparing crosscutting practices in STEM disciplines. Science \& Education, 29(4), 949-979. https://doi.org/10.1007/s11191-020-00147-1

Drake S. M., y Reid, J. L. (2020). 21st Century competencies in light of the history of integrated curriculum. Frontiers in Education, 5, Artículo 122. https://doi.org/10.3389/feduc.2020.00122

Espinal, L. M., y Silveira, F. (2019). La generación de prácticas, proyectos o programas en educación STEM-STEAM en el marco de una diplomatura virtual paraAmérica Latina. En B. Macedo, S. Silveira, M. García Astete, D. Meziat y L. Bengochea (Eds.), Enseñanza y aprendizaje de las ciencias en debate (pp. 622-631). Universidad de Alcalá.

European Commission. (2007). Science education now: a renewed pedagogy for the future of Europe. European Communities.

Greca, I. M., Ortiz-Revilla, J., y Arriassecq, I. (2021). Diseño y evaluación de una secuencia de enseñanza-aprendizaje STEAM para Educación Primaria. Revista Eureka sobre Enseñanza

\footnotetext{
${ }^{1}$ Las referencias marcadas con un asterisco indican los estudios incluidos en la revisión.
} 
y Divulgación de las Ciencias, 18(1), 1802. http://doi.org/10.25267/Rev_Eureka_ensen_ divulg_cienc.2021.v18.i1.1802

García-Carrillo, C., Greca, I. M., y Fernández-Hawrylak, M. (2021). Teacher perspectives on teaching the STEM approach to educational coding and robotics in primary education. Educational Sciences, 11(2), Artículo 64. https://doi.org/10.3390/educsci11020064

Gresnigt, R., Taconis, R., van Keulen, H., Gravemeijer, K., y Baartman, L. (2014). Promoting science and technology in primary education: a review of integrated curricula. Studies in Science Education, 50(1), 47-84. https://doi.org/10.1080/03057267.2013.877694

Herro, D., y Quigley, C. (2017). Exploring teachers' perceptions of STEAM teaching through professional development: implications for teacher educators. Professional Development in Education, 43(3), 416-438. https://doi.org/10.1080/19415257.2016.1205507

Kang, N-H. (2019). A review of the effect of integrated STEM or STEAM (science, technology, engineering, arts, and mathematics) education in South Korea. Asia-Pacific Science Education, 5(6), 1-22. https://doi.org/10.1186/s41029-019-0034-y

Kelley, T. R., y Knowles, J. G. (2016). Aconceptual framework for integrated STEM education. International Journal of STEM Education, 3(11), 1-11. https://doi.org/10.1186/s40594-016-0046-z

*Kim, P. W. (2016). The wheel model of STEAM education based on traditional Korean scientific contents. Eurasia Journal of Mathematics, Science \& Technology Education, 12(9), 23532371. https://doi.org/10.12973/eurasia.2016.1263a

*Kim, H., y Chae, D-H. (2016). The development and application of a STEAM programbased on traditional Korean culture. Eurasia Journal of Mathematics, Science \& Technology Education, 12(7), 1925-1936. https://doi.org/10.12973/eurasia.2016.1539a

Korea Foundation for the Advancement of Science and Creativity. (2012). Policy directions of STEAM education: introductory training of KOFAC STEAM. Foundation for the Advancement of Science and Creativity.

*Kummanee, J., Nilsook, P., y Wannapiroon, P. (2020). Digital learning ecosystem involving steam gamification for a vocational innovator. International Journal of Information and Education Technology, 10(7), 533-539. https://doi.org/10.18178/ijiet.2020.10.7.1420

Kwan, R., y Wong, B. T-M. (2021). Latest advances in STEAM education research and practice: a review of the literature. International Journal of Innovation and Learning, 29(3), 323-339. https://doi.org/10.1504/IJIL.2021.114528

*Lin, C-L., y Tsai, C-Y. (2021). The effect of a pedagogical STEAM model on students' project competence and learning motivation. Journal of Science Education and Technology, 30(1), 112-124. https://doi.org/10.1007/s10956-020-09885-x

Little, T. (2012). 21st Century learning and progressive education: an intersection. International Journal of Progressive Education, 8(3), 1-9. https://bit.ly/39mAKOA

Martín-Páez, T., Aguilera, D., Perales-Palacios, F. J., y Vílchez-González, J. M. (2019). What are we talking about when we talk about STEM education? A review of literature. Science Education, 103(4), 799-822. https://doi.org/10.1002/sce.21522

McComas, W. F., y Burgin, S. R. (2020). A critique of "STEM" education. Science \& Education, 29(4), 805-829. https://doi.org/10.1007/s11191-020-00138-2

Millar, V. (2020). Trends, issues and possibilities for an interdisciplinary STEM curriculum. Science \& Education, 29(4), 929-948. https://doi.org/10.1007/s11191-020-00144-4

Moher, D., Liberati, A., Tetzlaff, J., y Altman, D. G. (2009). Preferred reporting items for systematic reviews and meta-analyses: The PRISMA Statement. PLoS Med, 6(7), 1-6. https://doi. org/10.1371/journal.pmed.1000097

National Research Council. (2014). STEM Integration in K-12 education. Status, prospects, and an agenda for research. The National Academies Press.

Ortiz-Revilla, J. (2020). El desarrollo competencial en la Educación Primaria: efectos de una propuesta STEAM integrada [Tesis doctoral, Universidad de Burgos]. Repositorio Institucional de la Universidad de Burgos. https://bit.ly/39mMWii 
Ortiz-Revilla, J., Adúriz-Bravo, A., y Greca, I. M. (2020). A framework for epistemological discussion around an integrated STEM education. Science \& Education, 29(4), 857-880. https://doi. org/10.1007/s11191-020-00131-9

Ortiz-Revilla, J., Greca, I. M., y Adúriz-Bravo, A. (2018). La Educación STEAM y el desarrollo competencial en la Educación Primaria. En I. M. Greca y J. Á. Meneses Villagrá (Eds.), Proyectos STEAM para la Educación Primaria. Fundamentos y aplicaciones prácticas (pp. 41-54). Dextra.

Ortiz-Revilla, J., Greca, I. M., y Arriassecq, I. (2021). A theoretical framework for integrated STEM education. Science \& Educacion. Publicación anticipada en línea. https://doi.org/10.1007/ s11191-021-00242-x

Ortiz-Revilla, J., Greca, I. M., y Meneses-Villagrá, J. Á. (2021). Efectos de una propuesta STEAM integrada en el desarrollo competencial del alumnado de Educación Primaria. Infancia y Aprendizaje. Publicación anticipada en línea. https://doi.org/10.1080/02103702.2021. 1925473

Osborne, J., y Dillon, J. (2008). Science education in Europe: critical reflections. The Nuffield Foundation.

Quigley, C. F., y Herro, D. (2016). "Finding the joy in the unknown": implementation of STEAM teaching practices in middle school science and math classrooms. Journal of Science Education and Technology, 25(3), 410-426. https://doi.org/10.1007/s10956-016-9602-z

*Quigley, C., Herro, D., y Jamil, F. M. (2017). Developing a conceptual model of STEAM teaching practices. School Science and Mathematics, 117(1-2), 1-12. https://doi.org/10.1111/ ssm.12201

Reynante, B. M., Selbach-Allen, M. E., y Pimentel, D. R. (2020). Exploring the promises and perils of integrated STEM through disciplinary practices and epistemologies. Science \& Education, 29(4), 785-803. https://doi.org/10.1007/s11191-020-00121-x

Ritz, J. M., y Fan, S-C. (2015). STEM and technology education: international state of the art. International Journal of Technology and Design Education, 25(4), 429-451. https://doi. org/10.1007/s10798-014-9290-z

Romero-Ariza, M. (2017). El aprendizaje por indagación: ¿existen suficientes evidencias sobres sus beneficios en la enseñanza de las ciencias? Revista Eureka sobre Enseñanza y Divulgación de las Ciencias, 27(2), 286-299. https://bit.ly/3Cy1ylm

Sanders, M. (2008). STEM, STEM education, STEMmania. The Technology Teacher, 68(4), 20-26. https://bit.ly/39qlQqE

Teixeira, E. S., Greca, I. M., y Freire, O. (2012). The History and Philosophy of Science in physics teaching: a research synthesis of didactic interventions. Science\&Education, 21(6), 771796. http://doi.org/10.1007/s11191-009-9217-3

*Trott, C. D., Even, T. L., y Frame, S. M. (2020). Merging the arts and sciences for collaborative sustainability action: a methodological framework. Sustainability Science, 15(4), 10671085. https://doi.org/10.1007/s11625-020-00798-7

*Wannapiroon, N., y Petsangsri, S. (2020). Effects of STEAMification model in flipped classroom learning environment on creative thinking and creative innovation. TEM Journal, 9(4), 1647-1655. https://doi.org/10.18421/TEM94-42

Yakman, G. (2008). ST $\Sigma @ M$ education: an overview of creating a model of integrative education [Paper presentation]. ITEA 2008 Annual Conference, Salt Lake City, UT, Estados Unidos.

Zeidler, D. L. (2016). STEM education: a deficit framework for the twenty first century? A sociocultural socioscientific response. Cultural Studies of Science Education, 11(1), 11-26. https://doi. org/10.1007/s11422-014-9578-z

Zeidler, D. L., Sadler, T. D., Simmons, M. L., y Howes, E. V. (2005). Beyond STS: a research based framework for socio-scientific issues education. Science Education, 89(3), 357-377. https:// doi.org/10.1002/sce.20048

\section{Cómo citar en APA:}

Ortiz-Revilla, J., Sanz-Camarero, R. y Greca, I. M. (2021). Una mirada crítica a los modelos teóricos sobre educación STEAM integrada. Revista Iberoamericana de Educación, 87(2), 13-33.

https://doi.org/10.35362/rie8724634 